Domínguez-Alonso, J.; López-Castelo, A. y Portela-Pino, I. (2018) Propiedades psicométricas del autoinforme de barreras para la práctica del ejercicio físico (ABPEF) / Psychometric Properties of the Barrier Autoinform for the Practice of the Physical Exercise (ABPEF). Revista Internacional de Medicina y Ciencias de la Actividad Física y el Deporte vol. 18 (72) pp. 753-768 Http://cdeporte.rediris.es/revista/revista72/artpropiedades979.htm

DOI: http://doi.org/10.15366/rimcafd2018.72.010

\title{
ORIGINAL
}

\section{PROPIEDADES PSICOMÉTRICAS DEL AUTOINFORME DE BARRERAS PARA LA PRÁCTICA DEL EJERCICIO FÍSICO (ABPEF)}

\section{PSYCHOMETRIC PROPERTIES OF THE BARRIER AUTOINFORM FOR THE PRACTICE OF THE PHYSICAL EXERCISE (ABPEF)}

\author{
Domínguez-Alonso, J.' ; López-Castelo, A. ${ }^{2}$ y Portela-Pino, l. ${ }^{3}$ \\ 1 Doctor en Psicopedagogía, Universidad de Vigo (España) jdalonso@uvigo.es \\ 2 Doctor en Psicología, Universidad de Vigo (España) alopez@uvigo.es \\ ${ }^{3}$ Master en Condicionantes Genéticos, Nutricionales y Ambientales del Crecimiento y \\ Desarrollo, Universidad de Vigo (España) iportela@uvigo.es
}

Código UNESCO / UNESCO code: 6199 Otras especialidades psicológicas: Psicología del deporte/ Others: Sport Psychology

Clasificación Consejo de Europa / Council of Europe classification: 15 Psicología del deporte/ Sport Psychology

Recibido 30 de enero de 2017 Received January 30, 2017

Aceptado 17 de febrero de 2017 Accepted February 17, 2017

\section{RESUMEN}

El estudio consistió en examinar las propiedades psicométricas del cuestionario de autoinforme de barreras para la práctica del ejercicio físico (ABPEF) y comprobar el efecto del género sobre dichas barreras. Se han estudiado sus propiedades psicométricas con una muestra de 342 adolescentes (media de edad: 15.02 \pm 1.86 ). Se realizaron análisis de confiabilidad, análisis factorial exploratorio, correlación ítem con puntaje total y análisis factorial confirmatorio. Se encuentra una buena confiabilidad interna $(\alpha=.86)$, adecuada correlación ítem con puntaje total, y la presencia de cuatro factores en el análisis factorial exploratorio con correlaciones significativas entre las mismas. El análisis 
factorial confirmatorio mostró adecuado ajuste a los datos de un modelo tetrafactorial del cuestionario ( $\mathrm{X} 2$ /gI = 3.696; GFI=.89; AGFI=.86; RMSEA=.061). El género femenino presenta mayores barreras para la práctica del ejercicio físico. Este cuestionario es un instrumento fiable y válido y se sugiere su uso en futuras investigaciones.

PALABRAS CLAVE: Ejercicio físico, barreras, propiedades psicométricas, adolescencia, ABPEF.

\section{ABSTRACT}

The objective of this study was to examine the psychometric properties of the self-report questionnaire for physical exercise (ABPEF). And to chek the effect of gender on those barriers. Their psychometric properties were studied with a sample of 342 adolescents (mean age: $15.02 \pm 1.86$ ). We performed reliability analysis, exploratory factor analysis, item correlation with total score and confirmatory factor analysis. We found a good internal reliability $(\alpha=.86)$, an adequate item correlation with total score, and the presence of four factors in the exploratory factor analysis with significant correlations between them. The confirmatory factor analysis showed adequate adjustment to the data of a tetrafactorial model of the questionnaire $(\mathrm{X} / \mathrm{gl}=3.696$; $\mathrm{GFI}=.89$; $\mathrm{AGFI}=.86$; RMSEA = .061). The female gender presents greater barriers to the practice of physical exercise. This questionnaire is a reliable and valid instrument for the population under study, and its use in future research is suggested.

KEY WORDS: Physical exercise, barriers, psychometric properties, adolescence, ABPEF.

\section{INTRODUCCIÓN}

La importancia de la actividad física para la salud está avalada por numerosos estudios que así lo constatan (Brown, 2005; Dishman, Health \& Lee, 2008; Powell, Paluch \& Blair, 2011; Wilmot et al., 2012). De hecho, los beneficios de la práctica de la actividad física moderada a vigorosa durante la infancia y la adolescencia incluyen un menor riesgo cardiometabólico, menos masa grasa y una mayor aptitud física, especialmente cardiorrespiratoria (Dobbins, Husson, DeCorby \& LaRocca, 2013).

A nivel mundial, se estima que la inactividad física causa entre el $6 \%$ y el $10 \%$ de las principales enfermedades no transmisibles como la enfermedad coronaria, la diabetes tipo 2 y los cánceres de mama y colón. Además, este comportamiento malsano causa el $9 \%$ de la mortalidad prematura, o más de 5.3 de los 57 millones de muertes en 2008 (Lee et al., 2012). 
No obstante, aunque se considera que la actividad física en la adolescencia aumenta la probabilidad de convertirse en un adulto activo y saludable, y reduce el riesgo de futuros problemas de salud (Uijtdewilligen et al., 2011), el sedentarismo está aumentando en todos los países (Janssen \& Leblanc, 2010; Lee et al., 2012) hasta el punto de considerar la propia inactividad como un factor de riesgo para las enfermedades modernas no transmisibles (Danaei et al., 2009; Hallal et al., 2012; Li \& Siegrist, 2012; Park, Lee, Kang, Rhee \& Park, 2012). Es más, la actividad física disminuye durante la adolescencia en ambos sexos pero fundamentalmente en las chicas (Langguth et al., 2015).

En general, dada la importancia en la determinación de barreras que dificultan la realización de la actividad física, se han realizado estudios en países tan diversos como Estados Unidos, Australia, Japón, Brasil, Singapur, Malasia o España dado que en todos ellos las barreras personales, ambientales o sociales percibidas se asocian inversamente con el nivel de actividad física. Por tanto, el análisis de las barreras resulta importante no solo para poder evitarlas sino también porque la percepción de estas barreras se asocia con una mayor prevalencia de inactividad física en el tiempo libre de los adolescentes (Dias, Loch \& Ronque, 2015).

Sin embargo, las barreras y el grado de su asociación con la inactividad física dependen de la población estudiada (Ibrahim, Karim, Lai Oon \& Wan Ngah, 2013), no encontrando apenas estudios que puedan explicar la relación entre el comportamiento hacia la práctica de la actividad física y los modelos cognitivos sociales (Plotnikoff, Costigan, Karunamuni \& Lubans, 2013). Así pues, entre las barreras más importantes aparecen la falta de tiempo, la percepción de que otras actividades recreativas con la familia y los amigos son más divertidas, la falta de disciplina, el coste de algunas actividades, el no poder disfrutarlas con otras personas, la falta de motivación o la falta de instalaciones. Entre las menos citadas están la percepción de que la actividad física no es beneficiosa para la salud y la sensación de vergüenza (Justine, Azizan, Hassan, Salleh \& Manaf, 2013; Kimberly Hultquist \& McLester, 2013; Reichert, Barros, Domingues \& Hallal, 2007; Zabinski, Saelens, Stein, Hayden-Wade \& Wilfley, 2003). Resultan influencias significativas también la influencia del profesor de educación física como modelo y la influencia social de los amigos (Serra-Puyal, ZaragozaCasterad \& Generelo-Lanaspa, 2014).

El interés por el estudio de las barreras que impiden la práctica del ejercicio físico, se originó en los años 90 al comenzar a utilizar instrumentos para la medición de barreras, dado que disponer de un instrumento valido, fiable y aplicable en diferentes poblaciones resulta de máximo interés para la generalización y posible comparación de resultados. Surgen así, trabajos que analizan el tema utilizando cuestionarios con preguntas directas sobre la percepción o no de determinadas barreras, mientras otros se decantan por 
escalas tipo lickert (Allison, Dwyer \& Makin, 1999; Arzu, Handan, Tuzun \& Eker, 2006; Costa \& McCrae, 1992; Cheng et al., 2003; Kenneth et al., 2005; Rauh, Hovell, Hofdtetter, Sallis \& Gleghorn, 1992; Robbins, Pender \& Kazanis, 2003; USDHHS, 1999; Vasudevan, Rimmer \& Kviz, 2015).

Entre los instrumentos más utilizados figuran el Perceived Barriers Questionnaire (PBQ) de O'Neill y Reid (1991), el San Diego Health and Exercise Questionnaire (SDHEQ; Rauh, Hovell, Hofdtetter, Sallis y Gleghorn, 1992), el cuestionario Barriers to Being Active Quiz (BBAQ) del United States Department of Human Health Services (USDHHS,1999), el Kinesiophobia Causes Scale (KCS) questionnaire de Knapik, Saulicz y Gnat 2011; el Exercise Benefits/Barriers Scale (EBBS) de Sechrist, Walker y Pender (1987) validada para adultos por Brown (2005) o el BPAQ-MI para personas con discapacidad validado por Vasudevan, Rimmer y Kviz, 2015).

Una de las pocas aportaciones realizadas en lengua castellana es el Autoinforme de Barreras para la Práctica de Ejercicio Físico (ABPEF), propuesto inicialmente por Capdevila (2005) y adaptado por Niñerola, Capdevila y Pintanel (2006). En consecuencia, el objetivo de este trabajo es evaluar las propiedades psicométricas del ABPEF (en la versión castellana de 17 ítems) en poblaciones adolescentes, centrándose en la fiabilidad y validez, además de comprobar la influencia del género en dichas barreras.

\section{MÉTODO}

\section{Participantes}

La muestra estuvo conformada por 342 adolescentes de la Comunidad Autónoma de Galicia. Se utilizó como marco poblacional base, los datos y cifras de la enseñanza no universitaria (Xunta de Galicia, 2015). De esta manera, la muestra se distribuyó de la siguiente manera: un $45.9 \%$ de los adolescentes encuestados son mujeres y un 54.1\% son hombres. Además, un 26.9\% tiene 12 o 13 años, un $24.6 \%$ tiene 14 o 15 años, y un 48.5\% tiene 16 o 17 años. La media de edad fue de $15.1(\mathrm{DE}=1.86)$

\section{Instrumento}

Para analizar las barreras o dificultades percibidas en relación al ejercicio físico se ha utilizado un cuestionario elaborado "ad hoc" con datos personales (edad y género), y el Autoinforme de Barreras para la Práctica del Ejercicio Físico (ABPEF; Capdevila, 2005), adaptado por Niñerola, Capdevila y Pintanel (2006). Esta versión consta de 17 ítems que se responden en una escala Likert de 0 (razón poco probable que me impide practicar ejercicio físico en próximas semanas) a 10 (razón muy probable que me impide practicar ejercicio físico) 
puntos (Anexo I). El estudio original informa de cuatro subescalas diferentes (imagen corporal/ansiedad física y social; fatiga/pereza; obligaciones/falta de tiempo; ambiente/instalaciones) con una buena fiabilidad y adecuada validez.

\section{Procedimiento}

El cuestionario se administró de manera colectiva en estudiantes de educación secundaria obligatoria en horario regular de clases, durante el curso 2015-16. Después de comunicar las instrucciones oportunas y previo consentimiento informado (centro y familias), todo el alumnado cumplimentó de forma voluntaria la información solicitada. Se cumplieron los protocolos éticos de investigación con especial énfasis en la confidencialidad.

\section{Análisis de los datos}

Para el análisis de los datos se emplearon el programa estadístico SPSS 21 y el programa AMOS 21. Se llevó a cabo, en primer lugar, un análisis descriptivo de los ítems, media, desviación típica, correlación elemento-total corregida, Alpha de Cronbach si se elimina un elemento, así como los índices de asimetría y curtosis para evaluar el comportamiento normal de las variables. A continuación, se realizó un análisis factorial exploratorio (varimáx con káiser), con el fin de comprobar la estructura factorial inicial. Después, se correlacionaron los factores obtenidos y se estimó un análisis factorial confirmatorio (método de estimación de mínimos cuadrados no ponderados) para el modelo de cuatro factores. Finalmente, se valoró la consistencia interna de los indicadores, mediante el estadístico alfa de Cronbach.

\section{RESULTADOS}

\section{Estadísticos descriptivos: análisis de la calidad psicométrica de los ítems}

Con el fin de evaluar la distribución de la normalidad en los reactivos del cuestionario, se llevó a cabo un análisis de los ítems (tabla 1), mostrando medias homogéneas con desviaciones típicas alejadas de cero, lo cual indica una discriminación de los mismos. Asimismo, muestran correlaciones ítem-total corregidas positivas y superiores a .30 , indicando que todos contribuyen a calcular lo que mide el test y en la misma dirección. Ello también es refrendado por el análisis del Alfa de Cronbach si se elimina un elemento, evidenciando que la supresión de cualquiera de los ítems no mejoraría la fiabilidad del conjunto del cuestionario $(\alpha=.863)$. Finalmente, los valores de asimetría fueron inferiores a 2 y curtosis inferiores a 7 en todos los casos, cumpliendo los criterios de normalidad. En consecuencia, los ítems que conforman el cuestionario ABPEF siguen un comportamiento normal en todos sus reactivos. 
Rev.int.med.cienc.act.fís.deporte - vol. 18 - número 72 - ISSN: 1577-0354

Tabla 1. Estadísticos descriptivos para los ítems del cuestionario ABPEF

\begin{tabular}{|c|c|c|c|c|c|c|}
\hline & $M$ & $\mathrm{DE}$ & $\begin{array}{l}\text { Correlación } \\
\text { elemento- } \\
\text { total } \\
\text { corregida }\end{array}$ & $\begin{array}{l}\text { Alfa de } \\
\text { Cronbach } \\
\text { si se } \\
\text { elimina el } \\
\text { elemento }\end{array}$ & $\begin{array}{l}\text { Asimetria } \\
(E T=.132)\end{array}$ & $\begin{array}{l}\text { Curtosis } \\
(E T=.263)\end{array}$ \\
\hline ABPF1 & 2.30 & 1.91 & .354 & .851 & 1.81 & 3.22 \\
\hline ABPF2 & 3.83 & 2.94 & .465 & .847 & .834 & -.497 \\
\hline ABPF3 & 1.80 & 1.82 & .449 & .847 & 1.76 & 6.43 \\
\hline ABPF4 & 4.67 & 3.02 & .355 & .850 & .311 & -1.14 \\
\hline ABPF5 & 2.71 & 2.30 & .331 & .852 & 1.50 & 1.59 \\
\hline ABPF6 & 2.25 & 2.19 & .623 & .839 & 1.92 & 2.94 \\
\hline ABPF7 & 2.61 & 2.22 & .445 & .847 & 1.43 & 1.35 \\
\hline ABPF8 & 2.25 & 2.11 & .658 & .838 & 1.86 & 2.82 \\
\hline ABPF9 & 3.13 & 2.70 & .578 & .840 & 1.17 & .308 \\
\hline ABPF10 & 2.50 & 2.58 & .644 & .837 & 1.71 & 1.72 \\
\hline ABPF11 & 3.79 & 2.91 & .367 & .853 & .758 & -.682 \\
\hline ABPF12 & 2.58 & 2.35 & .486 & .845 & 1.63 & 1.80 \\
\hline ABPF13 & 2.25 & 2.31 & .593 & .840 & 1.03 & 3.20 \\
\hline ABPF14 & 2.16 & 1.92 & .365 & .850 & 1.86 & 3.08 \\
\hline ABPF15 & 1.86 & 1.75 & .544 & .844 & 1.59 & 6.97 \\
\hline ABPF16 & 2.09 & 2.18 & .598 & .840 & 1.30 & 4.55 \\
\hline ABPF17 & 1.83 & 1.74 & .339 & .851 & 1.73 & 6.91 \\
\hline
\end{tabular}

\section{Propiedades psicométricas del ABPEF}

Para evaluar la validez de constructo del cuestionario ABPEF en la muestra seleccionada en este estudio, se realizó, primero, un Análisis Factorial Exploratorio (AFE). Previamente, los índices de Kaiser-Meyer-Olkin (KMO) y de esfericidad de Bartlett han demostrado ser adecuados para ejecutar el AFE $(\mathrm{KMO}=.85$; Bartlett $=2170.64 ; p<.001)$. Así pues, se llevó a cabo el AFE por el Método de Componentes Principales, con una rotación varimax para obtener la estructura más simple y coherente (se han recogido únicamente los pesos factoriales superiores a .40). Los resultados muestran que los diecisiete ítems del cuestionario se agrupan en cuatro factores: imagen corporal/ansiedad físicasocial (los valores de las cargas factoriales aparecen en un rango entre .634 y .864); fatiga/pereza (valores de las cargas factoriales en un rango entre .422 y .767); obligaciones/falta de tiempo (valores de las cargas factoriales en un rango entre .637 y .866); $y$, ambiente/instalaciones (con valores de las cargas factoriales en un rango entre .421 y .712). Dichos factores explican un $59.12 \%$ de la varianza total del instrumento (tabla 2). Asimismo, todos los ítems presentaron comunalidades mayores al $40 \%$. 
Tabla 2. Matriz de componentes rotados, comunalidad y varianza explicada por los factores del ABPEF tras la rotación con la muestra $(n=342)$

\begin{tabular}{|c|c|c|c|c|c|}
\hline ITEMS & IC/AFS & $\mathrm{F} / \mathrm{P}$ & $\mathrm{O} / \mathrm{FT}$ & $\mathrm{A} / \mathrm{I}$ & $h^{2}$ \\
\hline ABPEF13 & .864 & & & & .499 \\
\hline ABPEF16 & .761 & & & & .667 \\
\hline ABPEF6 & .755 & & & & .488 \\
\hline ABPEF10 & .748 & & & & .690 \\
\hline ABPEF15 & .721 & & & & .502 \\
\hline ABPEF3 & .634 & & & & .647 \\
\hline ABPEF2 & & .767 & & & .568 \\
\hline ABPEF9 & & .697 & & & .584 \\
\hline ABPEF1 & & .679 & & & .617 \\
\hline ABPEF8 & & .460 & & & .642 \\
\hline ABPEF5 & & .449 & & & .773 \\
\hline ABPEF12 & & .422 & & & .532 \\
\hline ABPEF11 & & & .866 & & .761 \\
\hline ABPEF4 & & & .827 & & .456 \\
\hline ABPEF7 & & & .637 & & .577 \\
\hline ABPEF17 & & & & .712 & .641 \\
\hline ABPEF14 & & & & .421 & .606 \\
\hline Varianza Explicada & $24.01 \%$ & $13.79 \%$ & $12.42 \%$ & $8.88 \%$ & $59.11 \%$ \\
\hline
\end{tabular}

Fuente: Elaboración propia. Método de extracción: Análisis de componentes principales. Método de rotación: Normalización Varimax con Kaiser

A continuación, se analizó la validez convergente mediante el coeficiente de correlación de Pearson, para comprobar la relación entre las puntuaciones de los factores del cuestionario ABPEF (tabla 3). Se observaron correlaciones significativas $(p<.01)$ entre los cuatro factores, con valores moderados en fatiga/pereza-imagen corporal/ansiedad física 0 social $(r=.531)$ y ambiente/instalaciones-imagen corporal/ansiedad física o social ( $r=.409)$, y bajos en ambiente/instalaciones-fatiga/pereza ( $r=346)$, obligaciones/falta de tiempofatiga/pereza ( $r=.292)$, ambiente/instalaciones-obligaciones/falta de tiempo $(r=.241)$, y obligaciones/falta de tiempo-imagen corporal/ansiedad física o social $(r=.206)$. 
Tabla 3. Análisis correlacional de los factores del cuestionario ABPEF

\begin{tabular}{|c|c|c|c|c|}
\hline & $\begin{array}{l}\text { Imagen corporal } \\
\text { Ansiedad física- } \\
\text { social }\end{array}$ & $\begin{array}{l}\text { Fatiga } \\
\text { Pereza }\end{array}$ & $\begin{array}{l}\text { Obligaciones } \\
\text { Falta de tiempo }\end{array}$ & $\begin{array}{l}\text { Ambiente } \\
\text { Instalaciones }\end{array}$ \\
\hline $\begin{array}{l}\text { Imagen corporal } \\
\text { Ansiedad física- } \\
\text { social }\end{array}$ & - & & & \\
\hline $\begin{array}{l}\text { Fatiga } \\
\text { Pereza }\end{array}$ & $.531^{\star *}$ & - & & \\
\hline $\begin{array}{l}\text { Obligaciones } \\
\text { Falta de tiempo }\end{array}$ & $.206^{\star *}$ & $.292^{* *}$ & - & \\
\hline $\begin{array}{l}\text { Ambiente } \\
\text { Instalaciones }\end{array}$ & $.409^{* *}$ & $.346^{* *}$ & $.241^{\star \star}$ & - \\
\hline
\end{tabular}

En último lugar, el análisis factorial confirmatorio (método de estimación por máxima verosimilitud) de la versión del cuestionario de 17 ítems y cuatro factores presenta resultados satisfactorios (figura 1). Las cargas factoriales en los cuatro factores oscilan entre .30 y .86 (consideradas aceptables) y las saturaciones factoriales estandarizadas se encuentran entre moderadas (.40) y altas (.74). Así, las cargas factoriales promediaron: en imagen corporal/ansiedad físicasocial .73 (rango .60 a .84); en fatiga/pereza .58 (rango .40 a .72); en obligaciones/falta de tiempo .69 (rango .54 a .86); y en ambiente/instalaciones .44 (rango .44 a .45). Además, imagen corporal/ansiedad física-social presenta una fuerte correlación con ambiente/instalaciones $(r=.74)$ y fatiga/pereza $(r=$ .68). La fatiga pereza tiene fuerte correlación con ambiente/instalaciones ( $r=$ .71). Del mismo modo, se muestran bajas correlaciones entre: obligaciones/falta de tiempo con ambiente/instalaciones $(r=.42)$; obligaciones/falta de tiempo con fatiga/pereza $(r=.37)$; e imagen corporal/ansiedad física-social con obligaciones/falta de tiempo $(r=.31)$. 


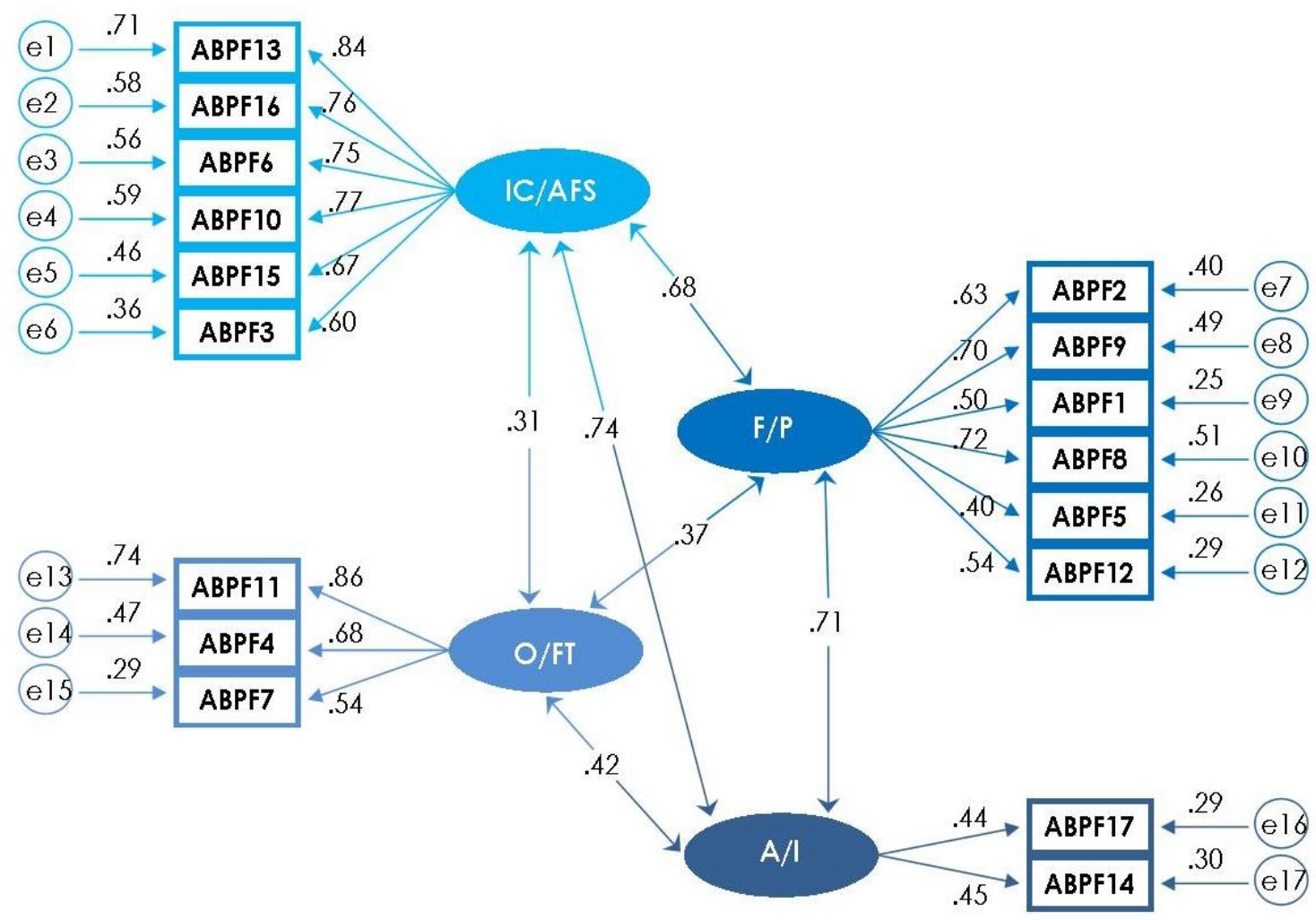

Figura 1. Coeficientes de regresión y saturaciones factoriales estandarizadas del cuestionario ABPEF (M4F)

Finalmente, se pone a prueba la hipótesis de factor único y de cuatro factores. Los resultados no ajustan a un modelo unidimiensional $(x 2=757.941$; $\mathrm{gl}=119 ; \mathrm{x} 2 \mathrm{gl}=6.369 ; \mathrm{GFI}=.77 ; \mathrm{AGFI}=.71 ; \mathrm{RMSEA}=.125)$, pero evidencian un ajuste aceptable al modelo de cuatro factores: $\mathrm{X} 2=417.612 ; \mathrm{gl}=113 ; \mathrm{X} 2 \mathrm{gl}=$ 3.696; $\mathrm{GFI}=.89 ; \mathrm{AGFI}=.86$; $\mathrm{RMSEA}=.061$.

\section{Confiabilidad del cuestionario}

El alpha de Cronbach para el cuestionario ABPEF es de .86 para la muestra completa, el cual es considerado como muy aceptable. Además, los valores obtenidos para los cuatro factores, se situaron por encima del .70. Concretamente: imagen corporal/ansiedad física-social ( $\alpha=.87$ ), fatiga/pereza $(\alpha=.76)$; obligaciones/falta de tiempo ( $\alpha=.73)$; ambiente/instalaciones $(\alpha=.72)$.

\section{Efectos de interacción del género}

Se realizó estadístico t-student (Tabla 4) con la muestra, donde se encontraron, en relación con el género, diferencias significativas ( $t_{341}=11.65, p$ $<$.001), concentrándose estas diferencias en los factores imagen corporal/ansiedad física-social $\left(\mathrm{t}_{341}=11.65, p<.001\right)$, fatiga/pereza $\left(\mathrm{t}_{341}=11.65\right.$, 
$p<.001)$ y ambiente/instalaciones ( $\left.\mathrm{t}_{341}=11.65, p<.001\right)$. Estas diferencias daban una mayor presencia de barreras para la práctica del ejercicio físico a favor de las chicas $(M=17.67, M=10.21, M=10.09$, respectivamente) frente a los chicos ( $\mathrm{M}=12.72, \mathrm{M}=8.44, \mathrm{M}=8.59$, respectivamente). Así pues, la mayor percepción de las barreras es en el género femenino $(M=49.86)$.

Tabla 4. Estadísticos descriptivos y análisis de las diferencias en función del género

\begin{tabular}{|c|c|c|c|c|c|}
\hline FACTOR del ABPEF & GÉNERO & $\mathbf{M}$ & DT & $t$ & $p$ \\
\hline \multirow{2}{*}{$\begin{array}{l}\text { Imagen corporal/ } \\
\text { Ansiedad física social }\end{array}$} & Masculino & 12.72 & 9.45 & \multirow{2}{*}{24.59} & \multirow{2}{*}{$<.001$} \\
\hline & Femenino & 17.67 & 13.11 & & \\
\hline \multirow{2}{*}{ Fatiga/Pereza } & Masculino & 8.44 & 5.41 & \multirow{2}{*}{13.07} & \multirow[t]{2}{*}{$<.001$} \\
\hline & Femenino & 10.21 & 6.57 & & \\
\hline \multirow{2}{*}{$\begin{array}{l}\text { Obligaciones/Falta } \\
\text { tiempo }\end{array}$} & Masculino & 10.39 & 6.37 & \multirow{2}{*}{1.28} & \multirow{2}{*}{.258} \\
\hline & Femenino & 11.87 & 6.81 & & \\
\hline \multirow{2}{*}{ Ambiente/Instalaciones } & Masculino & 8.59 & 4.91 & \multirow{2}{*}{8.36} & \multirow{2}{*}{$<.001$} \\
\hline & Femenino & 10.09 & 5.76 & & \\
\hline \multirow{2}{*}{$\begin{array}{l}\text { Puntuacion total } \\
\text { (17 ítems) }\end{array}$} & Masculino & 40.15 & 19.09 & \multirow{2}{*}{11.65} & \multirow{2}{*}{$<.001$} \\
\hline & Femenino & 49.86 & 23.37 & & \\
\hline
\end{tabular}

\section{DISCUSIÓN}

Este estudio se propuso evaluar las propiedades psicométricas de la versión de autoinforme de las barreras para la práctica del ejercicio físico (ABPEF-17). En términos generales, los resultados de validez y confiabilidad indican que es un instrumento adecuado para valorar las barreras o impedimentos que dificultan a los adolescentes la práctica de ejercicio físico. Aunque son recomendables estudios posteriores, los resultados hallados son coincidentes con otros trabajos realizados (Niñerola, Capdevila \& Pintanel, 2006; Reigal, Videra, Márquez \& Parra, 2013; Samperio, Jiménez-Castuera, Lobato, Leyton \& Claver, 2016).

En primer lugar, se busca confirmar la estructura obtenida mostrando las correlaciones ítem-total corregidas positivas (Clark \& Watson, 2003) y superiores a .30 (Nunnally \& Bernstein, 1995), lo cual indica que todos los ítems contribuyen a calcular lo que mide el autoinforme y en la misma dirección. Además, presenta un comportamiento normal en todos sus reactivos, ya que los valores de asimetría fueron inferiores a 2 y curtosis inferiores a 7 en todos los casos, cumpliendo los criterios de normalidad (Curran, West \& Finch, 1996).

En segundo término, los índices de Kaiser-Meyer-Olkin (KMO) y de esfericidad de Bartlett han demostrado ser adecuados para ejecutar el AFE (Lloret-Segura, FerreresTraver, Hernández-Baeza \& Tomás-Marco, 2014). En cuanto a la estructura factorial, se encontraron cuatro dimensiones: imagen corporal/ansiedad física-social, fatiga/pereza, obligaciones/falta de tiempo, y ambiente/instalaciones. Los cuatro factores explicaron el $59.1 \%$ de la varianza total de las barreras para la práctica del ejercicio físico. Así, la cantidad de dimensiones resulta semejante a las señaladas en la versión adaptada de Niñerola, Capdevila \& Pintanel (2006). Sin embargo, uno de los ítems cargó en un factor 
distinto al propuesto en dicha versión adaptada (el ítem 15 Encontrarme a disgusto con la gente que hace ejercicio conmigo desaparece del factor "ambiente/instalaciones" y pasa a formar parte del factor "imagen corporal/ansiedad física-social"). A pesar de ello, la estructura resultó teóricamente coherente, por lo que se respetó la pertenencia de los ítems a las dimensiones aquí observadas.

A continuación, la correlación entre los cuatro factores (validez convergente) resultó significativa $(p<.01)$, positiva y moderada. De forma adicional, el AFC demostró que las cuatro variables latentes conforman el constructo de barreras que dificultan la práctica del ejercicio físico, como se encontró en el análisis factorial exploratorio. Finalmente, se pone a prueba la hipótesis de factor único y de cuatro factores. Los resultados no ajustan a un modelo unidimensional, pero evidencian un ajuste aceptable al modelo de cuatro factores según los criterios de interpretación establecidos por Browne y Cudeck (1993), Hu y Bentler (1999), o Byrne (2001). En conjunto, los resultados obtenidos por medio del análisis factorial (exploratorio y confirmatorio) nos informan de la validez de la estructura hipotetizada, aportándose indicaciones de un ajuste adecuado del modelo de cuatro factores. La consistencia interna del autoinforme global ( $\alpha$ : .86) y de los cuatro factores (oscila entre $\alpha: .72$ y $\alpha: .86)$ es buena, superando sensiblemente el valor recomendado de ser siempre mayor a .70 (Nunnally \& Berstein, 1995).

En relación al género, las mujeres presentan mayores barreras en la práctica del ejercicio físico, lo cual indica que sus niveles de actividad física son inferiores (Casado, Alonso, Hernández \& Jiménez, 2009; Fairclough \& Stratton, 2006; Serra-Puyal, Generelo-Lanaspa \& Zaragoza-Casterad, 2010; Beltrán-Carrillo, Devís-Devís \& PeiróVelert, 2012; Langguth et al., 2015). Estos datos refuerzan los trabajos de Cordente (2006), Fairclough \& Stratton (2006), Peiró, Devís, Beltrán \& Fox (2008), Barquero, Barriopedro \& Montil (2008), Martínez-Gómez et al. (2009), que coinciden en señalar a los hombres son más activos que las mujeres en el periodo adolescente.

\section{CONCLUSIONES}

Los resultados observados en el presente estudio determinan que el ABPEF muestra indicadores suficientes para ser considerado válido y fiable y poder ser utilizado en el estudio y análisis de las barreras que impiden o dificultan la práctica del ejercicio físico en el periodo adolescente. El conocimiento de dichas barreras puede ser relevante tanto para el diagnóstico como para la definición de áreas prioritarias de intervención, en aquellos individuos en riesgo de abandonar la práctica físico-deportiva en la adolescencia, etapa en la que existe un descuido considerable de dicha práctica. En cuanto a la variable género, los hombres y mujeres presentaron diferencias significativas en todas las barreras para la práctica del ejercicio físico, poniendo de manifiesto que las mujeres perciben con mayor frecuencia e intensidad las barreras que impiden 0 dificultan la práctica del ejercicio físico (excepto en el factor obligaciones/falta de tiempo).

Como principales limitaciones del estudio indicar el uso exclusivo de los datos del autoinforme para la evaluación de los diferentes factores, con los posibles sesgos y restricciones que ello conlleva, y la falta de criterios externos que proporcionen el estudio 
de la validez convergente de dicho cuestionario. Así pues, son necesarias más investigaciones que traten de corroborar los resultados obtenidos en diferentes poblaciones y contextos, utilizando distintos análisis estadísticos, ya que la validación y fiabilidad de un instrumento no se puede limitar a un solo estudio.

\section{REFERENCIAS BIBLIOGRÁFICAS}

Allison, K.R., Dwyer, J.M., \& Makin, S. (1999). Perceived Barriers to Physical Activity among High School Students. Preventive Medicine, 28(6), 608-615. https://doi.org/10.1006/pmed.1999.0489

Arzu, D., Handan, E., \& Eker, L. (2006). Perceived Barriers to Physical Activity in University Students. Journal of Sports Science and Medicine, 05, 615-620.

Barquero, P., Barriopedro, M.I., \& Montil, M. (2008). Patrones de actividad física en niños con sobrepeso y normopeso: un estudio de validez concurrente. Apunts: Medicina De l'Esport, 43(159), 127-134. https://doi.org/10.1016/S1886-6581(08)70088-4

Beltrán-Carrillo, V.J., Devís-Devís, J., \& Peiró-Velert, C. (2012). Actividad física y sedentarismo en adolescentes de la Comunidad Valenciana. Revista Internacional de Medicina y Ciencias de la Actividad Física y el Deporte, 12(45), 122-137.

Brown, S.A. (2005). Measuring Perceived Benefits and Perceived Barriers for Physical Activity. Am J Health Behav, 29(2), 107-116. https://doi.org/10.5993/AJHB.29.2.2

Browne, M.W., \& Cudeck, R. (1993). Alternative ways of assessing model fit. In: K. A. Bollen \& J. S. Long (Eds.), Testing structural equation models (pp. 136-162). Beverly Hills, CA: Sage.

Byrne, B.M. (2001). Structural equation modeling with AMOS: Basic concepts, applications, and programming. Mahwah, NJ: Lawrence Erlbaum.

Capdevila, L. (2005). Actividad física y Salud. Barcelona: Miracle.

Casado, C., Alonso, N., Hernández, V., \& Jiménez, R. (2009). Actividad física en niños españoles: factores asociados y evolución 2003-2006. Revista Pediatría de Atención Primaria, 11(42), 219-231.

Cheng, K.Y., Cheng, P.G., Mak, K.T., Wong, S.H., Wong, Y.K., \& Yeung, E.W. (2003). Relationships of perceived benefits and barriers to physical activity, physical activity participation and physical fitness in Hong Kong female adolescents. Journal of Sports Medicine Physical Fitness, 43, 523-529.

Clark, L.A., \& Watson, D. (2003). Constructing validity: Basic issues in objective scale development. In A. E. Kazdin (Ed.), Methodological issues \& strategies in clinical research (3ª ed., pp. 207-231). Washington: APA.

Cordente, C.A. (2006). Estudio epidemiológico del nivel de actividad física y de otros parámetros de interés relacionados con la salud bio-psico-social de los alumnos de ESO del municipio de Madrid (Tesis doctoral). Universidad de Castilla-La Mancha, España.

Costa, P.T., \& McCrae, R.R. (1992). Revised NEO Personality Inventory (NEO-PI-R) and NEO Five-Factor Inventory (NEO-FFI) professional manual. Odessa, FL: Psychological Assessment Resources.

Curran, P.J., West, S.G., \& Finch, J.F. (1996). The robustness of test statistics to nonnormality and specification error in confirmatory factor analysis. Psychological Methods, 1(1), 16-29. https://doi.org/10.1037/1082-989X.1.1.16

Danaei, G.D.E., Mozaffarian, D., Taylor, B., Rehm, J., Murray, C.J., \& Ezzati, M. (2009). The preventable causes of death in the United States: Comparative risk 
assessment of dietary, lifestyle, and metabolic risk factors. PLoS Med 6(4), e1000058. https://doi.org/10.1371/journal.pmed.1000058

De Rezende, L.F., Azeredo, C.M., Canella, D.S., Claro, R.M., De Castro, I.R., Levy, R.B., \& Luiz, O. (2014). Sociodemographic and behavioral factors associated with physical activity in Brazilian adolescents. BMC Public Health, 14, 485. https://doi.org/10.1186/1471-2458-14-485

Dias, D.F., Loch, M.R., \& Ronque, E.V. (2015). Perceived barriers to leisure-time Physical activity and associated factors in adolescents. Ciencia \& Saude Coletiva, 20(11), 3339-3350. https://doi.org10.1590/1413-812320152011.00592014

Dishman R., Health G., \& Lee I.M: (2008). Physical Activity Epidemiology. Champaign, IL: Human Kinetics.

Dobbins M., Husson H., DeCorby K., \& LaRocca R.L. (2013). School-based physical activity programs for promoting physical activity and fitness in children and adolescents aged 6 to 18. Cochrane Database of Systematic Reviews, Issue 2. Art. No.: CD007651. https://doi.org/10.1002/14651858.CD007651.pub2

Fairclough, S.J., \& Stratton, G. (2006). A review of physical activity levels during elementary school physical education. Journal of Teaching in Physical Education, 25, 240-258. https://doi.org/10.1123/jtpe.25.2.240

Hallal, P.C., Andersen, L.B., Bull, F.C., Guthold, R., Haskell, W., \& Ekelund, U. (2012). Global physical activity levels: surveillance progress, pitfalls, and prospects. The Lancet, 380, 247-257. https://doi.org/10.1016/S0140-6736(12)60646-1

Hu, L.T., \& Bentler, P.M. (1999). Cut-off criteria for fit indexes in covariance structure analysis. Conventional criteria versus new alternatives. Structural Equation Modeling, 6, 1-55. https://doi.org/10.1080/10705519909540118

Ibrahim, S., Karim, N.A., Lai-Oon, N. \& Wan-Ngah, W.Z. (2013). Perceived physical activity barriers related to body weight status and sociodemographic factors among Malaysian men in Klang Valley. BMC Public Health, 13, 275. https://doi.org/10.1186/1471-2458-13-275

Jansen, I., \& Leblanc, A.G. (2010). Systematic review of the health benefits of physical activity and fitness in school-aged children and youth. International Journal of Behavioral Nutrition and Physical Activity, 7(40), 2-16. https://doi.org/10.1186/1479-5868-7-40.

Justine, M., Azizan, A., Hassan, V., Salleh, Z., \& Manaf, H. (2013). Barriers to participation in physical activity and exercise among middle-aged and elderly individuals. Singapore Med J, 54(10), 581-586. https://doi.org/10.11622/smedj.2013203

Kenneth, R.A., Dwyer, J.M., Goldenberg, E., Fein, A., Yoshida, K.K., \& Boutilier, M. (2005). Male adolescents' reasons for participating in physical activity, barriers to participation and suggestions for increasing participation. Adolescence, 40, 155170.

Kimberly, K.M.S., Hultquist, C.N. \& McLester, Y.R. (2013). A Comparison of Motivational Factors and Barriers to Physical Activity Among Traditional Versus Nontraditional College Students. Journal of American College Health, 61 (2), 60-66. https://doi.org/10.1080/07448481.2012.753890

Knapik, A., Saulicz, E., \& Gnat, R. (2011). Kinesiophobia. Introducing a New Diagnostic Tool. J Hum Kinet., 28, 25-31. https://doi.org/10.2478/v10078-011-0019-8 
Langguth, N., Könen, T., Matulis, S., Steil, R., Gawrilow, C., \& Stadler, G. (2015). Barriers to Physical Activity in Adolescents. A Multidimensional Approach. Zeitschrift für Gesundheitspsychologie, 23, 47-59. https://doi.org/10.1026/0943-8149/a000136

Lee, M., Shiroma, E., Lobelo, F., Puska, P., Blair, S., \& Katzmarzyk, P. (2012). Effect of physical inactivity on major non-communicable diseases worldwide: an analysis of burden of disease and life expectancy. The Lancet, 380, 219-229. https://doi.org/10.1016/S0140-6736(12)61031-9

Li, A. \& Siegrist, J. (2012). Physical Activity and Risk of Cardiovascular Disease: A MetaAnalysis of Prospective Cohort Studies International Journal of Environmental Research and Public Health, 9(2), 391-407. https://doi.org/10.3390/ijerph9020391

Lloret-Segura, S., Ferreres-Traver, A., Hernández-Baeza, A., \& Tomás-Marco, I. (2014). El análisis factorial exploratorio de los ítems: una guía práctica, revisada y actualizada. Anales de Psicología, 30(3), 1151-1169. https://doi.org/10.6018/analesps.30.3.199361

Martínez-Gómez, D., Martínez-De-Haro, V., Del-Campo, J., Zapatera, B., Welk, G.J, Villagra, A.M., \& Veiga, O.L. (2009). Validez de cuatro cuestionarios para valorar la actividad física en adolescentes españoles. Gaceta Sanitaria, 23(6), 512-517. https://doi.org/10.1016/j.gaceta.2009.02.013

Niñerola i Maymí, J., Capdevila-Ortís L., \& Pintanel-Bassets, M. (2006). Barreras percibidas y actividad física: el autoinforme de barreras para la práctica de ejercicio físico. Revista de Psicología del Deporte, 15(1), 53-69.

Nunnally, J.C., \& Bernstein, I.J. (1995). Psychometric theory. Madrid: McGraw-Hill.

O'neill, K., \& Reid, G., 1991: Perceived barriers to physical activity by older adults. Canadian Journal of Public Health, 82(6), 392-396.

Park, S., Lee, J., Kang, D.Y., Rhee, C.W., \& Park, B.J. (2012). Indoor Physical Activity Reduces All-Cause and Cardiovascular Disease Mortality Among Elderly Women. Journal of Preventive Medicine and Public Health, 45(1), 21-28. https://doi.org/10.3961/jpmph.2012.45.1.21

Peiró, C., Devís, J., Beltrán., V.J., \& Fox, K.R. (2008). Variability of Spanish adolescents' physical activity patterns by seasonality, day of the week and demographic factors. European Journal of Sport Science, 8(3), 161-173.

Plotnikoff, R.C., Costigan, S.A., Karunamuni, N., \& Lubans, D.R. (2013). Social cognitive theories used to explain physical activity behavior in adolescents: A systematic review and meta-analysis. Preventive Medicine, 5685, 245-253. https://doi.org/10.1016/j.ypmed.2013.01.013

Powell, K., Paluch, A., \& Blair, S. (2011). Physical Activity for Health: What Kind? How Much? How Intense? On Top of What? Annual Review of Public Health, 32, 349365. https://doi.org/10.1146/annurev-publhealth-031210-101151

Rauh, M.J.D., Hovell, M.F., Hofstetter, C.R., Sallis, J.F., \& Gleghorn A. (1992). Reliability and Validity of Self-Reported Physical-Activity in Latinos. International Journal of Epidemiology, 21, 966-971. https://doi.org/10.1093/ije/21.5.966

Reichert, F.F., Barros, A.J.D., Domingues, M.R., \& Hallal, P.C. (2007). The Role of Perceived Personal Barriers to Engagement in Leisure-Time Physical Activity. American Journal of Public Health, 97(3), 515-519. https://doi.org/10.2105/AJPH.2005.070144

Reigal, R.E., Videra, A., Marquez, M.V., \& Parra, J.L. (2013). Autoconcepto físico multidimensional y barreras para la práctica física en la adolescencia. Apunts. 
Rev.int.med.cienc.act.fís.deporte - vol. 18 - número 72 - ISSN: 1577-0354

Educación Fisica y Deportes, 111, 23-28. https://doi.org/10.5672/apunts.20140983.es.(2013/1).111.02

Robbins, L.B., Pender, N.J., \& Kazanis, A.S. (2003). Barriers to Physical Activity Perceived by Adolescent Girls Pages 206-212 May 2003. J Midwifery Womens Health, 48 (3), 206-212. https://doi.org/10.1016/S1526-9523(03)00054-0

Samperio, J., Jiménez-Castuera, R., Lobato, S., Leyton, M., \& Claver, F. (2016). Variables motivacionales predictoras de las barreras para la práctica de ejercicio físico en adolescentes. Cuadernos de Psicología del Deporte, 16(2), 65-76.

Sechrist, K.R., Walker, S.N., \& Pender, NJ. (1987). Development and psychometric evaluation of the Exercise Benefits/Barriers Scale. Research in Nursing \& Health, 10, 357-365. https://doi.org/10.1002/nur.4770100603

Serra-Puyal, J.R., Generelo-Lanaspa, E., \& Zaragoza-Casterad, J. (2010). Barreras para la realización de actividad física en adolescentes en la provincia de Huesca. Revista Internacional de Medicina y Ciencias de la Actividad Física y el Deporte, 10 (39), 470-482.

Serra-Puyal, J.R., Zaragoza-Casterad, J., \& Generelo-Lanaspa, E. (2014). Influencias de "otros significativos" para la práctica de actividad física en adolescentes. Revista Internacional de Medicina y Ciencias de la Actividad Física y el Deporte, 14 (56), 735-753

U.S. Department of Health and Human Services, Public Health Service, Centers for Disease Control and Prevention, National Center for Chronic Disease Prevention and Health Promotion, Division of Nutrition and Physical Activity. (1999). Barriers to Being Active Quiz. In: Promoting Physical Activity: A Guide for Community Action (100-101). Champaign, IL: Human Kinetics.

Uijtdewilligen, L., Singh, A.S., Twisk, J.W.R., Koppes, L.L., Van Mechelen, W., \& Chinapaw, M.J. (2011). Adolescent predictors of objectively measured physical activity and sedentary behaviour at age 42: the Amsterdam Growth and Health Longitudinal Study (AGAHLS). Int $J$ Behav Nutr Phys Act., 8, 1-5. https://doi.org/10.1186/1479-5868-8-1

Vasudevan, V., Rimmer, J., \& Kviz, F. (2015). Development of the Barriers to Physical Activity Questionnaire for People with Mobility Impairments. Disability and Health Journal, 8(4). 547-556. https://doi.org/10.1016/j.dhjo.2015.04.007

Wilmot, E.G., Edwardson, C.L., Achana, F.A., Davies, M.J., Gorely, T., Gray L.J... Biddle, S.J.H. (2012). Sedentary time in adults and the association with diabetes, cardiovascular disease and death: systematic review and meta-analysis. Diabetologia, 55, 2895-2905. https://doi.org/10.1007/s00125-012-2677-z

Xunta de Galicia (2015). Datos e cifras do ensino non universitario: curso 2015-

16. Santiago de Compostela: Consellería de Cultura, Educación e Ordenación Universitaria.

Zabinski, M.F., Saelens, B.E., Stein, R.I., Hayden-Wade, H.A., \& Wilfley, D.E. (2003). Overweight Children's Barriers to and Support for Physical Activity. Obesity Research, 11, 238-246. https://doi.org/10.1038/oby.2003.37

Número de citas totales / Total references: 53 (100\%)

Número de citas propias de la revista / Journal's own references: $03(5,6 \%)$

Rev.int.med.cienc.act.fís.deporte - vol. 18 - número 72 - ISSN: 1577-0354 
ANEXO I. Autoinforme de barreras para la práctica de ejercicio físico

Instrucciones: Durante las próximas semanas ¿cuál es la probabilidad de que las siguientes razones te impidan realizar ejercicio físico?

\begin{tabular}{|c|c|c|c|c|c|c|c|c|c|c|}
\hline & \multicolumn{10}{|c|}{ Probabilidad } \\
\hline & \multicolumn{5}{|c|}{ Mucha } & \multicolumn{5}{|c|}{ Poca } \\
\hline $\begin{array}{c}\text { Razón que te impide practicar ejercicio físico las } \\
\text { próximas semanas }\end{array}$ & 1 & 2 & 3 & 4 & 5 & 6 & 7 & 8 & 9 & 10 \\
\hline $\begin{array}{l}\text { 1. Cansarme demasiado durante el ejercicio o miedo } \\
\text { a lesionarme }\end{array}$ & & & & & & & & & & \\
\hline 2. $\quad$ Tener pereza & & & & & & & & & & \\
\hline $\begin{array}{l}\text { 3. Sentir incomodidad por el aspecto que tengo con } \\
\text { ropa deportiva }\end{array}$ & & & & & & & & & & \\
\hline 4. Tener demasiado trabajo & & & & & & & & & & \\
\hline $\begin{array}{l}\text { 5. Tener "agujetas" o dolores musculares a } \\
\text { consecuencia del ejercicio }\end{array}$ & & & & & & & & & & \\
\hline 6. Sentir mi aspecto físico peor que el de los demás & & & & & & & & & & \\
\hline 7. Tener demasiadas obligaciones familiares & & & & & & & & & & \\
\hline 8. No estar "en forma" para practicar ejercicio & & & & & & & & & & \\
\hline 9. Faltar de voluntad para ser constante & & & & & & & & & & \\
\hline $\begin{array}{l}\text { 10. Pensar que la otra gente está en mejor forma que } \\
\text { yo }\end{array}$ & & & & & & & & & & \\
\hline 11. No encontrar el tiempo necesario para el ejercicio & & & & & & & & & & \\
\hline $\begin{array}{l}\text { 12. Notar cansancio o fatiga de forma habitual a lo largo } \\
\text { del día }\end{array}$ & & & & & & & & & & \\
\hline 13. Pensar que los demás juzgan mi apariencia física. & & & & & & & & & & \\
\hline $\begin{array}{l}\text { 14. Estar demasiado lejos del lugar donde puedo hacer } \\
\text { ejercicio }\end{array}$ & & & & & & & & & & \\
\hline $\begin{array}{l}\text { 15. Encontrarme a disgusto con la gente que hace } \\
\text { ejercicio conmigo }\end{array}$ & & & & & & & & & & \\
\hline $\begin{array}{l}\text { 16. Sentir vergüenza porque me están mirando } \\
\text { mientras hago ejercicio }\end{array}$ & & & & & & & & & & \\
\hline $\begin{array}{l}\text { 17. Que las instalaciones o los monitores no sean } \\
\text { adecuados }\end{array}$ & & & & & & & & & & \\
\hline
\end{tabular}

Rev.int.med.cienc.act.fís.deporte - vol. 18 - número 72 - ISSN: 1577-0354 